\title{
A note from the editor
}

(C) Macmillan Publishers Ltd., part of Springer Nature 2018

Recent scholarship draws attention to new Latino destinations as well as shifting demographics in long-established Latino communities across the United States. These studies have complicated our understanding of Latinidades and require that we more intentionally explore the dynamics of emerging and changing Latino spaces. Latino communities are never monolithic. Even in a context where one nationality predominates, variations of migration histories, generation, race, class, and language use exist. In addition, increasingly, there are more and more sites in which Latinos from different nationalities coexist. This reality suggests the need for more studies that help us understand intra-Latino relations in specific contexts.

This special issue focuses on Miami, a site with a long history of intra-Latino relations. As Sarah Mahler, this special issue's editor, points out, Miami is an ideal place to study intra-Latino relations since it is home to one of the largest concentrations of Latinos in the United States. In Miami, unlike in many other contexts, Latinos constitute a demographic majority; this is a site where interactions between established and emergent communities allow us to consider how Latinos relate to one another intra-ethnically. The authors included in this special issue are interested in exploring perceived similarities and differences among Latinos. Given that differences of migration processes, social contexts, political realities, patterns of racialization, immigration status, and other factors affect how Latinos of different nationalities view and interact with one another, the studies demonstrate points of both solidarity and distance among communities. This collection of articles reminds us again that solidarity among Latinos must be imagined and forged in each specific context rather than assumed to naturally exist. The issue is an invitation for more studies that offer a nuanced reading of intra-Latino relations. Clearly, focusing exclusively on pan-ethnic harmony or division reveals only a partial truth; a more comprehensive analysis includes both unity and discord and can best help us move toward building Latino solidarity across nationalities. 